\title{
Prader willi syndrome: global motor cognition and hypotonia
}

\author{
Larrivee $\mathbf{D}^{1,2 *}$ \\ ${ }^{1}$ Loyola University, Chicago, USA \\ ${ }^{2}$ Mind and Brain Institute, University of Navarra, Pamplona, Spain
}

\begin{abstract}
Among the central fetal hypotonia diseases, Prader Willi Syndrome (PWS) is distinguished for its profound, but transient motor lethargy in early infancy and the subsequent appearance of permanent hyperphagic and intellectual disability symptoms. Its relatively narrow and discretely defined etiology suggests that it may be empirically exploited to explore underlying motor processes. Current studies indicate that impaired neural correlates of task switching observed in its later stages are unlikely to contribute to early symptoms and may entail synaptogenic delays related to the formation of global motor planning circuitries.
\end{abstract}

\section{Motor Cognition in PWS}

Prader Willi syndrome is a neurodevelopmental disorder lacking the paternal expression of maternally imprinted genes at chromosomal locus 15q11-13. PWS patients present at birth with a severe hypotonia and show an initial failure to thrive, followed later by behavioral manifestations of hyperphagia and long term intellectual disability. Research to date implicates a combination of subcortical and higher order cortical structures, which underwrite features of reward processing, motivation, and affect resulting in abberant activity across broadly distributed neural networks [1]. How these neural impairments contribute to the severe hypotonia that is observed in young infants is unknown, a point that will be considered here.

Infant hypotonia is comprised of a wide number of etiological impairments that are challenging in diagnosis. Characterized by a generalized reduction in muscle tone, the problem of clinical polymorphism is further exacerbated by the non-specificity of clinical markers and nosological dependence [2]. Presenting symptoms of frog like posture, inability to resist gravitational inclines, and excessive joint mobility are observed in many infant neurological diseases [3]. Contributing hypotonia loci situate, for instance, in muscle (congenital myotonic dystrophy), neuromuscular junction (congenital myasthenia gravis), motor nerve (hypomyelinating neuropathy), motor soma of the spinal cord (spinal muscular atrophy), and in brain tissue (cerebral dysgenesis and metabolic diseases) [4]. Nearly 70 percent of all hypotonia cases, including Prader Willi Syndrome, trace their origin to etiologies affecting the central nervous system.

Differential diagnosis for PWS includes clinical evaluation, with algorithmic profiling like that of M Iraschi [5], that is now regularly combined with DNA and cytogenetic assays for confirmation. DNA based methylation within the critical chromosomal region detects, for example, nearly $99 \%$ of all PWS patients. PWS is symptomatically distinguished among the central etiologies by the requirement for tube feeding without coincident support for breathing, which is highly unusual for other central hypotonia origins, a finding that implicates cortical domains rather than brain stem autonomic activity.
The severity of hypotonia in PWS, its subsequent recovery after neonatal and infant stages, the persistence of behavioral and intellectual abnormalities, and its siting to intentional motor control suggest a unique and more narrowly defined etiology. Its appearance early in infancy is likely to relate to underlying impairments in central nervous system network connectivities that are ordinarily formed during major synaptogenic epochs, that is, during critical windows when synaptic connections for principal brain networks are being formed [6]. These heightened periods of plasticity entail a multiplicity of interacting factors including variation in genetic expression, responsivity to developmental cues, and enhanced sensitivity to environmental input, usually in the form of stimulus evoked activity [7]. As critical periods of development, these also constitute periods of increased vulnerability, when influencing factors may be less than optimal, or mis assimilated, leading to neurodevelopmental disorders [8]. Major periods are now known to encompass synaptogenesis in the brain stem (early), thalamus (intermediate), and cortical zones including the cerebellum (late).

For neurodevelopmental disorders occurring within these periods a frequently marked observation is a delay in the onset of synaptogenesis, which is then followed by a partial but permanent phase of recovery. Significantly, the displacement of cortical recovery resembles the time evolution of hypotonia that is exhibited in PWS patients, consistent with postulates that link PWS etiology to an impact on connectivity architecture occurring during this phase. For PWS, however, the persistence of other symptoms that can be traced to cortical origins suggests that some network architectures formed during these intervals are not linked to those that contribute to hypotonic symptoms. Hence, the differing symptomatic outcomes are likely to implicate distinct processes that are impacted by the disease in this crucial developmental stage.

Initial studies indicate that long term impairments may be related to executive mechanisms that reciprocally interact with the

${ }^{\star}$ Correspondence to: Denis Larrivee, Mind and Brain Institute, University of Navarra, Pamplona, Spain, E-mail: sallar1@aol.com

Received: June 13, 2018; Accepted: June 16, 2018; Published: June 18, 2018 
default mode network [9]. Individuals with PWS fail to show normal activity patterns related to task switching associated with task positive fronto parietal networks. In cued task switching tests, for example, there is a considerably reduced activation of the posterior parietal and ventromedial prefrontal cortices that is postulated to involve the attentional capacity of the default mode network.

The transient symptoms of PWS hypotonia, by contrast, are noted for their universal and profound character, and unlikely to entail neural correlates for task switching, that are more likely to involve regional oscillatory control and select motor responsivity [10]. The central origin of the etiology and the breadth of the tonic responses impacted, in fact, are telling signatures leading to the conclusion that hypotonia due to PWS involves aberrant activity across distributed cortical networks [1] potentially affecting basic mechanisms of motor output generation.

Knowledge of cortical motor processes has undergone something of a renaissance in the last decade and several are now invoked to explain the genesis of distributed network processes that control the skeletal musculature on a body wide basis [11]. This knowledge may be usefully explored alongside that of a generalized and profound hypotonia. Two of the more prominent schemes include 1) the use of processing primitives to coordinate spatiotemporal features of motor movement in distributed networks [12], and 2) motor planning processes that contextualize intentional movement to the whole individual and that involve sensory motor coupling [13].

\section{Processing Primitives}

Processing primitives entail basic physical properties that must be instantiated to relate motor movement to the events of the world, that is, they enable coordinated movements that are internally consistent and externally relevant for interactivity. Of these, timing, orientation, and their derivatives, like velocity and vectorial acceleration, are fundamental. As a functional principle information transmission within and between cortical regions needs to possess coincident, or at least directly relatable, coding strategies for communication efficiency. Implicitly, the generation of common coding schemes requires that mechanisms for accurate timing subserve the generation of codes, like those mediated by oscillatory or attractor like activity. Several lines of evidence suggest that their instantiation is chiefly of cerebellar origin.

First, the cerebellum is particularly noted for its stereotypical anatomical configuration, which is highly modular, consisting of cortical and subcortical zones [12]. In the cortical zone granule cells predominate, elaborating parallel fibers that activate all other neurons of the cortex. Parallel fibers form connections with the large Purkinje cells which project to the deep cerebellar nuclei. Cortical interneurons, including stellate, basket, and Lugaro cells, provide for feedforward excitation. Together these cells comprise a modular organization that is repeated throughout the cortical layer that is especially suited to the capturing of physical universals like space and time.

Second, timing hypotheses that premise a role for the cerebellum in the structuring of neural coding postulate that the cerebellum critically represents temporal relations between task associated events. Motor coordination, for instance, entails a precise spatiotemporal sequence that defines their vectorial trajectories [14]. Experiments of irregularity detection, notably, indicate that the cerebellum contributes to the processing of the stimuli [12]. Consistent with a timing role, moreover, fMRI studies indicate that velocity estimation is uniquely localized to the posterior cerebellum in lobule 7 [15].

Finally, among the cortical structures the cerebellum is traditionally associated with control of motor movement; the cerebellum, for example, projects to motor as well as to pre and supplementary motor areas. Recent studies reveal an even broader network of cerebellar connectivity. Cerebellar cortical loops, for example, establish connectivity networks with the thalamus, as well as the cerebrum to facilitate communication with motor sensory, parietal, and prefrontal zones known to participate in sensory motor coupling and executive functions.

If and how PWS may impact such mechanisms at this early stage of neural development remain for inferences, likely to be drawn from associations with broader mechanisms such as late epoch synaptogenesis for which a growing body of evidence is available, and focal fMRI classification methods during critical areas of timing emergence. Significantly, recent findings in postmortem Fragile X chromosome patients, a genetic aberration closely related to PWS, reveal anatomical deterioration in the cerebellum and hippocampus, structures engaged in generating timing and spatial primitives [16].

\section{Sensory Motor Coupling and Global Planning}

Conversely, there is now clear evidence that motor execution requires the formulation of action planning prior to execution and that such plans must be configured in the context of the whole individual. Several now classic studies on brain plasticity, in fact, show that specific sensory motor experiences involve a functional reorganization of the motor cortex in association with goal seeking. In human infants this cortical reorganization is closely related to the task that is learned and repeatedly practiced [17], like the use of one arm more than another that typically elicits larger upper limb representation in the contralateral brain hemisphere. Hence, the brains of these infants undergo continual connectivity reorganization as task learning proceeds.

A critical aspect of this mapping is its intentional performance, that is, actions that are intended for the individual as a whole with specific goals in mind. Together these results implicate the physical instantiation of network activity that links selected and selective actions to global performance. Without the instantiation of such a global representation, in fact, goal directed movements are not undertaken, a presupposition for explanations of results from Piaget's A not B task that relate to sensorimotor representations [13]. Brain mapping experiments using positron emission tomography (PET) or fMRI, for example, show the existence of identical cortical and subcortical networks activated during both motor imagery and action observation, networks that are directly concerned with motor execution [18]. In the case of Piaget's task these representations thus constitute motor images that have yet to be contextualized in development to a representation of the individual as a whole. For PWS infants, the breadth of impact on motor performance suggests a similar distortion of this generalized representation, a conclusion consistent with motor integration deficits in the PWS patient [19].

\section{Conclusion}

Fetal hypotonia comprises a broad spectrum of generalized motor impairments, nearly $70 \%$ of which are traced to central origins. Among these PWS is distinguished for its profound but transient expression of hypotonia with the subsequent appearance of long lasting hyperphagia and intellectual disability; hence, their temporal independence suggests the impairment of discrete neural correlates that are cortically localized. Faulty task switching that is manifest in later stages is unlikely to contribute to hypotonia, which may entail a more generalized impairment in motor planning. 


\section{References}

1. Manning KE, Holland AJ (2015) Puzzle Pieces: Neural Structure and Function in Prader-Willi Syndrome. Diseases 3: 382-415. [Crossref]

2. Prygunova TM, Radaeva TM, Stepanova EY (2015) Floppy infance syndrome: the impartance for the differential diagnosis of hereditary metabolic diseases and degenerative diseases of the nervous system. Current Pediatrics 14: 586-590.

3. Bodensteiner JB (2008) The evaluation of the hypotonic infant. Semin Pediatr Neurol 15: 10-20. [Crossref]

4. Hartley L, Ranjan R (2015) Evaluation of the floppy infant. Paediat Child Health 25: 498-505.

5. Igarashi M (2004) Floppy infant syndrome. J Clin Neuromuscul Dis 6: 69-90. [Crossref]

6. Ismail FY, Fatemi A, Johnston MV2 (2017) Cerebral plasticity: Windows of opportunity in the developing brain. Eur J Paediatr Neurol 21: 23-48. [Crossref]

7. Workman AD, Charvet CJ, Clancy B, Darlington RB, Finlay BL (2013). Modeling transformations of neurodevelopmental sequences across mammalian species. $J$ Neurosci 33: 7368-7383.

8. Meredith RM (2015) Sensitive and critical periods during neurotypical and aberrant neurodevelopment: a framework for neurodevelopmental disorders. Neuro Biobehav Rev 50: 180-188.

9. Woodcock KA, Humphreys GW, Oliver C, Hansen PC (2010) Neural correlates of task switching in paternal 15q11-q13 deletion Prader-Willi syndrome. Brain Res 1363: 128-142.
10. Buzsaki G, Draguhn A (2004) Neuronal oscillations in cortical networks. Science 304: 1926-1929. [Crossref]

11. Frak V, Nazir T, Goyette M, Cohen H, Jeannerod M (2010) Grip force is part of the semantic representation of manual action verbs. PLoS One 5: e9728. [Crossref]

12. D'Angelo E, Casali S (2013) Seeking a unified framework for cerebellar funtion and dysfunction: from circuit operations to cognition. Front Neural Circuits 6: 1-23.

13. Smith L (2009) Stability and flexibility in development. In: Spencer J, Thomas MSC, McClelland JL Toward a unified theory of development. Oxford University Press, Oxford.

14. Ivry RB, Spencer RM, Zelaznik HN, Diedrichsen J (2002) The cerebellum and event timing. Ann N Y Acad Sci 978: 302-317. [Crossref]

15. O'Reilly JX, Mesulam MM, Nobre AC (2008) The cerebellum predicts the timing of perceptual events. J Neurosci 28: 2252-2260. [Crossref]

16. Greco CM, Navarro CS, Hunsaker MR, Maezawa I, Shuler JF, et al. (2011) Neuropathologic features in the hippocampus and cerebellum of three older men with fragile X syndrome. Mol Autism 2: 2. [Crossref]

17. Corbetta D (2009) Brain, body, and mind: lessons from infant motor development In: Spencer J, Thomas MSC, McClelland JL (edn) Toward a unified theory of development. Oxford University Press, Oxford pp 51-56.

18. Jeannerod M (2005) Levels of representation of goal directed actions. In: Higher Order Motor Disorders. Freund HJ, Jeannerod M, Hallett M, and Leiguarda R (edn). Oxford: Oxford University Press, pps. 159-182.

19. Lo ST, Collin PJ, Hokken-Koelega AC (2015) Visual-motor integration in children with Prader-Willi syndrome. J Intellect Disabil Res 59: 827-834. [Crossref]

Copyright: $\odot 2018$ Larrivee D. This is an open-access article distributed under the terms of the Creative Commons Attribution License, which permits unrestricted use, distribution, and reproduction in any medium, provided the original author and source are credited. 